\section{IU⿴囗十⿺辶大 IUCrData}

ISSN 2414-3146

Received 12 February 2017

Accepted 13 February 2017

Edited by J. Simpson, University of Otago, New Zealand

Keywords: crystal structure; hydrogen bond; pyrazole.

\section{CCDC reference: 1532509}

Structural data: full structural data are available from iucrdata.iucr.org

\section{$\mathrm{N}$-\{2-[2-(5-Methyl-1H-pyrazol-3-yl)acetamido]- phenyl\}benzamide monohydrate}

\author{
Karim Chkirate, ${ }^{\mathrm{a} *}$ Joel T. Mague, ${ }^{\mathrm{b}}$ Nada Kheira Sebbar, ${ }^{\mathrm{a}}$ Younes Ouzidan ${ }^{\mathrm{c}}$ and \\ El Mokhtar Essassi ${ }^{\mathrm{a}}$
}

aLaboratoire de Chimie Organique Hétérocyclique, URAC 21, Pôle de Compétence Pharmacochimie, Av Ibn Battouta, BP 1014, Faculté des Sciences, Université Mohammed V, Rabat, Morocco, ${ }^{\mathbf{b}}$ Department of Chemistry, Tulane University, New Orleans, LA 70118, USA, and 'Laboratoire de Chimie Organique Appliquée, Université Sidi Mohamed Ben Abdallah, Faculté des Sciences et Techniques, Route d'Imouzzer, BP 2202, Fez, Morocco. *Correspondence e-mail: chkiratekarim@gmail.com

The asymmetric unit of the title compound, $\mathrm{C}_{19} \mathrm{H}_{18} \mathrm{~N}_{4} \mathrm{O}_{2} \cdot \mathrm{H}_{2} \mathrm{O}$, comprises the Ushaped pyrazole derivative and a solvent water molecule. The molecular conformation is partly determined by an intramolecular $\mathrm{N}-\mathrm{H} \cdots \mathrm{O}$ hydrogen bond. The crystal packing is directed by an extensive network of $\mathrm{O}-\mathrm{H} \cdots \mathrm{O}, \mathrm{N}-$ $\mathrm{H} \cdots \mathrm{O}, \mathrm{N}-\mathrm{H} \cdots \mathrm{N}$ and $\mathrm{C}-\mathrm{H} \cdots \mathrm{O}$ hydrogen bonds together with $\mathrm{C}-$ $\mathrm{H} \cdots \pi$ (ring) contacts that generate a three-dimensional network.
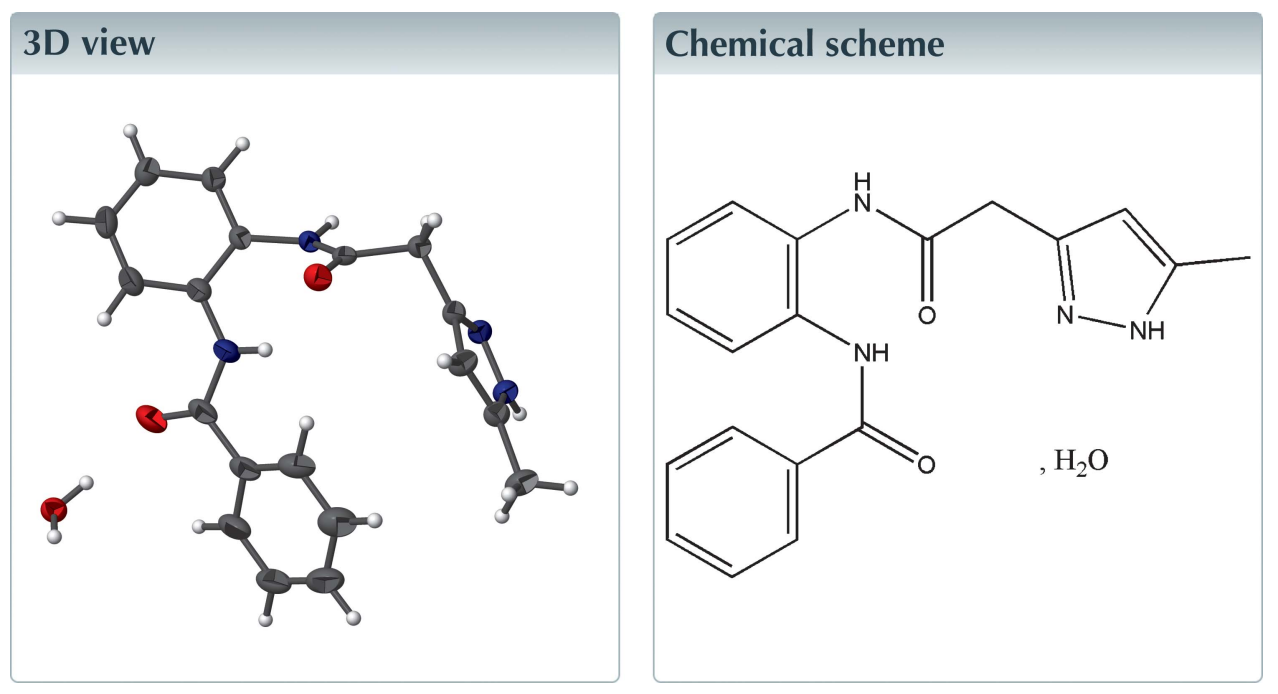

\section{Structure description}

Pyrazole derivatives have pharmacologically attractive biological applications (Havrylyuk et al., 2016) and interesting therapeutic properties (Khan et al., 2016). These compounds have been synthesized as target structures by many researchers and have been evaluated for their beneficial bioactivity and for the rational design of a new generation of small molecule drugs (Küçükgüzel \& Şenkardeş, 2015). Continuing our research in this field (Chkirate et al., 2001), we have synthesized $N$-2-benzamido-phenyl5-methyl-pyrazol-3-yl acetamide by reacting benzoyl chloride with $\mathrm{N}$-2-aminophenyl-5methyl-pyrazol-3-yl acetamide. The latter was obtained by the action of hydrazine on the 4-(oxopropylidene)-1,5-benzodiazepin-2-one (El Abbassi et al., 1989).

The title molecule adopts a U-shaped conformation due, in part, to the intramolecular $\mathrm{N} 1-\mathrm{H} 1 \cdots \mathrm{O} 2$ hydrogen bond (Table 1 and Fig. 1). The dihedral angle between the C1C6 and C8-C13 benzene rings is $49.67(5)^{\circ}$ while that between the latter ring and the pyrazole ring is $64.49(6)^{\circ}$. The packing is governed largely by a network of intermolecular hydrogen bonds including $\mathrm{N} 2-\mathrm{H} 2 A \cdots \mathrm{N} 3^{\mathrm{i}}, \quad \mathrm{N} 4-\mathrm{H} 4 A \cdots \mathrm{O} 3^{\mathrm{ii}}, \quad \mathrm{O} 3-$ 


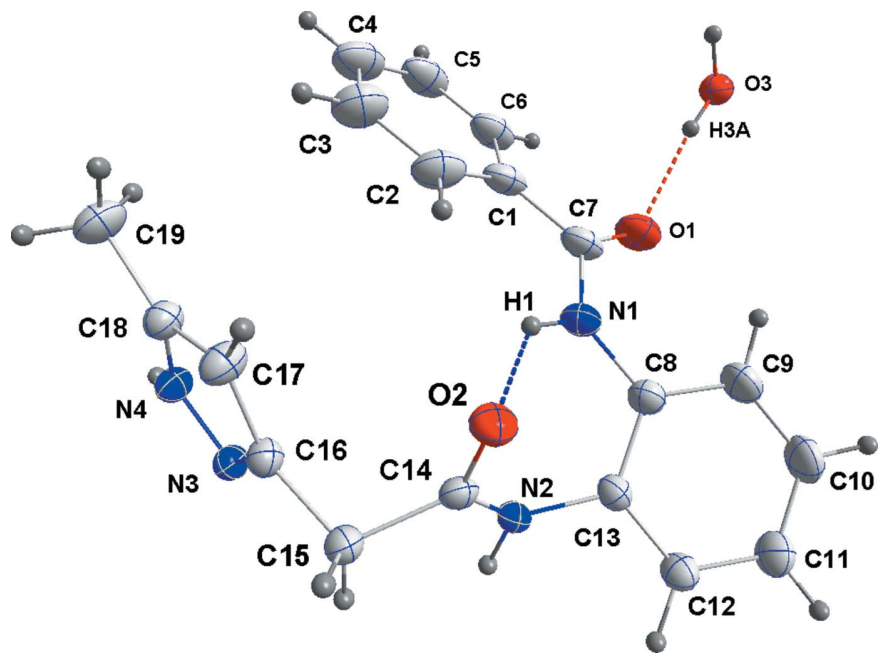

Figure 1

The asymmetric unit of the title compound, showing the atom-labelling scheme and $50 \%$ probability displacement ellipsoids. The intramolecular $\mathrm{N}-\mathrm{H} \cdots \mathrm{O}$ hydrogen bond is shown as a blue dashed line.

$\mathrm{H} 3 A \cdots \mathrm{O} 1, \mathrm{O} 3-\mathrm{H} 3 B \cdots \mathrm{O} 2^{\mathrm{iv}}$ and $\mathrm{C} 12-\mathrm{H} 12 \cdots \mathrm{O} 3^{\mathrm{iii}}$ (Table 1 and Fig. 2). In addition there are two $\mathrm{C}-\mathrm{H} \cdots \pi$ (ring) interactions: $\quad \mathrm{C} 15-\mathrm{H} 15 A \cdots \pi(\mathrm{C} 8-\mathrm{C} 13)^{\mathrm{v}}$ and $\mathrm{C} 19-$ $\mathrm{H} 19 A \cdots \pi(\mathrm{N} 3, \mathrm{~N} 4, \mathrm{C} 16-\mathrm{C} 18)^{\mathrm{vi}}$, Table 1 , that also contribute to the crystal packing. These contacts combine to generate a three-dimensional network, Fig. 2.

\section{Synthesis and crystallization}

To a solution of $5 \times 10^{-4} \mathrm{~mol}$ of $\mathrm{N}$-(2-aminophenyl-5-methylpyrazol-3-yl)acetamide dissolved in $10 \mathrm{ml}$ of ethanol was added $5 \times 10^{-4}$ mol of benzoyl chloride. The mixture was stirred for $24 \mathrm{~h}$ at room temperature. After filtration and recrystallization from ethanol, colourless single crystals were obtained with a yield of $66 \%$.

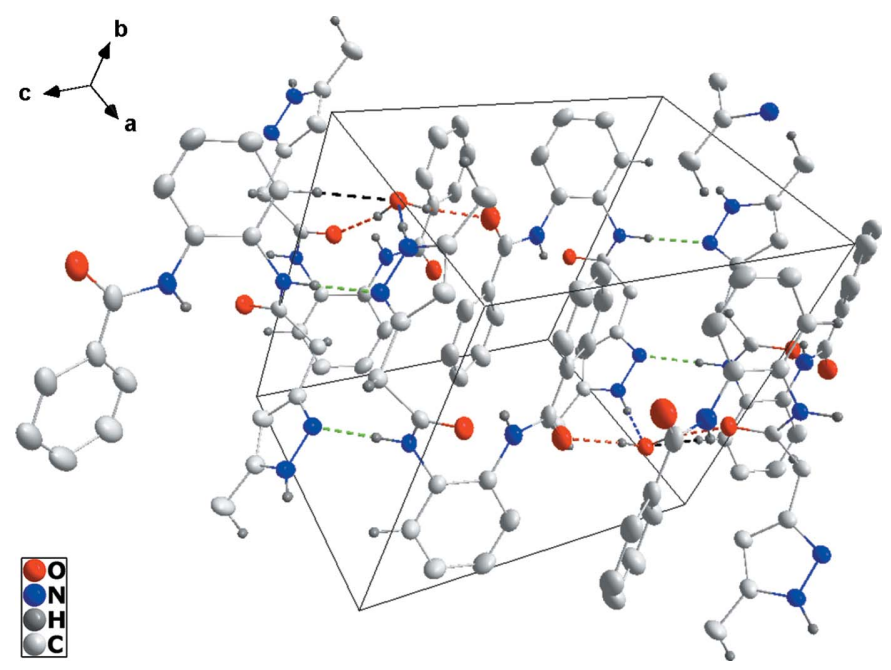

Figure 2

Crystal packing projected onto (111) with hydrogen bonds shown as dashed lines $[\mathrm{O}-\mathrm{H} \cdots \mathrm{O}$ (red); $\mathrm{N}-\mathrm{H} \cdots \mathrm{O}$ (blue); $\mathrm{N}-\mathrm{H} \cdots \mathrm{N}$ (green); $\mathrm{C}-$ $\mathrm{H} \cdots \mathrm{O}$ (black)].
Table 1

Hydrogen-bond geometry $\left(\AA,^{\circ}\right)$.

$\mathrm{Cg} 1$ and $\mathrm{Cg} 3$ are the centroids of the N3,N4,C16-C18 and $\mathrm{C} 8-\mathrm{C} 13$ rings respectively.

\begin{tabular}{lllll}
\hline$D-\mathrm{H} \cdots A$ & $D-\mathrm{H}$ & $\mathrm{H} \cdots A$ & $D \cdots A$ & $D-\mathrm{H} \cdots A$ \\
\hline $\mathrm{N} 1-\mathrm{H} 1 \cdots \mathrm{O} 2$ & $0.88(2)$ & $2.09(2)$ & $2.7902(17)$ & $135.0(19)$ \\
$\mathrm{N} 2-\mathrm{H} 2 A \cdots \mathrm{N} 3^{\mathrm{i}}$ & $0.95(2)$ & $1.98(2)$ & $2.9209(17)$ & $176.4(17)$ \\
$\mathrm{N} 4-\mathrm{H} 4 A \cdots 3^{\mathrm{ii}}$ & $0.92(2)$ & $1.88(2)$ & $2.7883(16)$ & $166.9(19)$ \\
$\mathrm{C} 12-\mathrm{H} 12 \cdots \mathrm{O} 3^{\mathrm{iii}}$ & $0.978(19)$ & $2.464(19)$ & $3.4042(19)$ & $161.2(14)$ \\
$\mathrm{O} 3-\mathrm{H} 3 A \cdots \mathrm{O} 1$ & $0.87(3)$ & $1.89(3)$ & $2.7445(16)$ & $167(2)$ \\
$\mathrm{O} 3-\mathrm{H} 3 B \cdots \mathrm{O} 2^{\text {iv }}$ & $0.94(3)$ & $1.83(3)$ & $2.7575(15)$ & $169(2)$ \\
$\mathrm{C} 15-\mathrm{H} 15 A \cdots C g 3^{\mathrm{v}}$ & $0.957(19)$ & $2.893(18)$ & $3.8485(15)$ & $174.0(14)$ \\
$\mathrm{C} 19-\mathrm{H} 19 A \cdots C g 1^{\text {vi }}$ & $0.98(3)$ & $2.86(3)$ & $3.645(2)$ & $138(2)$ \\
\hline
\end{tabular}

Symmetry codes: (i) $-x+1,-y+1,-z+2$; (ii) $-x+1,-y+1,-z+1$; $\quad$ (iii)

$x, y, z+1 ; \quad$ (iv) $\quad-x+2,-y+1,-z+1 ; \quad$ (v) $\quad-x+2,-y+1,-z+2$; $\quad$ (vi)

$-x+1,-y+2,-z+2$.

Table 2

Experimental details.

\begin{tabular}{ll}
\hline Crystal data & \\
Chemical formula & $\mathrm{C}_{19} \mathrm{H}_{18} \mathrm{~N}_{4} \mathrm{O}_{2} \cdot \mathrm{H}_{2} \mathrm{O}$ \\
$M_{\mathrm{r}}$ & 352.39 \\
Crystal system, space group & Triclinic, $P \overline{1}$ \\
Temperature $(\mathrm{K})$ & 150 \\
$a, b, c(\AA)$ & $8.4220(3), 10.0410(4), 10.8799(4)$ \\
$\alpha, \beta, \gamma\left({ }^{\circ}\right)$ & $101.889(2), 94.104(2), 90.402(1)$ \\
$V\left(\AA^{3}\right)$ & $897.79(6)$ \\
$Z$ & 2 \\
Radiation type & $\mathrm{Cu} \mathrm{K \alpha}$ \\
$\mu\left(\mathrm{mm}^{-1}\right)$ & 0.74 \\
Crystal size $(\mathrm{mm})$ & $0.15 \times 0.14 \times 0.10$ \\
& \\
Data collection & $\mathrm{Bruker} \mathrm{D} 8 \mathrm{VENTURE}$ PHOTON \\
Diffractometer & $100 \mathrm{CMOS}$ \\
& Multi-scan $(S A D A B S ;$ Bruker, \\
Absorption correction & $2016)$ \\
& $0.85,0.93$ \\
$T_{\text {min }}, T_{\text {max }}$ & $6831,3279,2846$ \\
No. of measured, independent and & \\
$\quad$ observed $[I>2 \sigma(I)]$ reflections & 0.028 \\
$R_{\text {int }}(\text { sin } \theta / \lambda)_{\text {max }}\left(\AA^{-1}\right)$ & 0.617 \\
& \\
Refinement & \\
$R\left[F^{2}>2 \sigma\left(F^{2}\right)\right], w R\left(F^{2}\right), S$ & $0.038,0.114,1.06$ \\
No. of reflections & 3279 \\
No. of parameters & 316 \\
$\mathrm{H}$-atom treatment & All H-atom parameters refined \\
$\Delta \rho_{\text {max }}, \Delta \rho_{\text {min }}\left(\mathrm{e} \AA^{-3}\right)$ & $0.20,-0.21$ \\
\hline
\end{tabular}

Computer programs: APEX3 and SAINT (Bruker, 2016), SHELXT (Sheldrick, 2015a), SHELXL2014 (Sheldrick, 2015b), DIAMOND (Brandenburg \& Putz, 2012) and SHELXTL (Sheldrick, 2008).

\section{Refinement}

Crystal and refinement details appear in Table 2. Eleven reflections appearing near the top of the frames on which they were recorded were omitted from the fineal refinement as they appeared to have been partially obscured by the nozzle of the low-temperature attachment.

\section{Acknowledgements}

The support of NSF-MRI Grant No. 1228232 for the purchase of the diffractometer and Tulane University for support of the Tulane Crystallography Laboratory are gratefully acknowledged. 


\section{References}

Brandenburg, K. \& Putz, H. (2012). DIAMOND. Crystal Impact GbR, Bonn, Germany.

Bruker (2016). APEX3, SAINT and SADABS. Bruker AXS Inc., Madison, Wisconsin, USA.

Chkirate, K., Regragui, R., Essassi, E. M. \& Pierrot, M. (2001). Z. Kristallogr. New Cryst. Struct. 216, 635-636.

El Abbassi, M., Djerrari, B., Essassi, E. M. \& Fifani, J. (1989). Tetrahedron Lett. 30, 7069-7070.
Havrylyuk, D., Roman, O. \& Lesyk, R. (2016). Eur. J. Med. Chem. 113, 145-166.

Khan, M. F., Alam, M. M., Verma, G., Akhtar, W., Akhter, M. \& Shaquiquzzaman, M. (2016). Eur. J. Med. Chem. 120, 170-201.

Küçükgüzel, Ş. G. \& Şenkardeş, S. (2015). Eur. J. Med. Chem. 1, 102110.

Sheldrick, G. M. (2008). Acta Cryst. A64, 112-122.

Sheldrick, G. M. (2015a). Acta Cryst. A71, 3-8.

Sheldrick, G. M. (2015b). Acta Cryst. C71, 3-8. 


\section{full crystallographic data}

IUCrData (2017). 2, x170251 [https://doi.org/10.1107/S2414314617002516]

\section{N-\{2-[2-(5-Methyl-1H-pyrazol-3-yl)acetamido]phenyl\}benzamide monohydrate}

Karim Chkirate, Joel T. Mague, Nada Kheira Sebbar, Younes Ouzidan and El Mokhtar Essassi

$N$-\{2-[2-(5-Methyl-1H-pyrazol-3-yl)acetamido]phenyl\}benzamide monohydrate

Crystal data

$\mathrm{C}_{19} \mathrm{H}_{18} \mathrm{~N}_{4} \mathrm{O}_{2} \cdot \mathrm{H}_{2} \mathrm{O}$

$M_{r}=352.39$

Triclinic, $P \overline{1}$

$a=8.4220(3) \AA$

$b=10.0410(4) \AA$

$c=10.8799$ (4) $\AA$

$\alpha=101.889(2)^{\circ}$

$\beta=94.104(2)^{\circ}$

$\gamma=90.402(1)^{\circ}$

$V=897.79(6) \AA^{3}$

Data collection

Bruker D8 VENTURE PHOTON 100 CMOS diffractometer

Radiation source: INCOATEC I $\mu \mathrm{S}$ micro-focus source

Mirror monochromator

Detector resolution: 10.4167 pixels $\mathrm{mm}^{-1}$

$\omega$ scans

Absorption correction: multi-scan

(SADABS; Bruker, 2016)

\section{Refinement}

Refinement on $F^{2}$

Least-squares matrix: full

$R\left[F^{2}>2 \sigma\left(F^{2}\right)\right]=0.038$

$w R\left(F^{2}\right)=0.114$

$S=1.06$

3279 reflections

316 parameters

0 restraints

Primary atom site location: structure-invariant direct methods
$Z=2$

$F(000)=372$

$D_{\mathrm{x}}=1.304 \mathrm{Mg} \mathrm{m}^{-3}$

$\mathrm{Cu} K \alpha$ radiation, $\lambda=1.54178 \AA$

Cell parameters from 5496 reflections

$\theta=4.5-71.9^{\circ}$

$\mu=0.74 \mathrm{~mm}^{-1}$

$T=150 \mathrm{~K}$

Block, colourless

$0.15 \times 0.14 \times 0.10 \mathrm{~mm}$

$T_{\min }=0.85, T_{\max }=0.93$

6831 measured reflections

3279 independent reflections

2846 reflections with $I>2 \sigma(I)$

$R_{\text {int }}=0.028$

$\theta_{\max }=72.2^{\circ}, \theta_{\min }=4.2^{\circ}$

$h=-10 \rightarrow 10$

$k=-11 \rightarrow 12$

$l=-12 \rightarrow 13$

Secondary atom site location: difference Fourier map

Hydrogen site location: difference Fourier map

All $\mathrm{H}$-atom parameters refined

$w=1 /\left[\sigma^{2}\left(F_{\mathrm{o}}^{2}\right)+(0.0641 P)^{2}+0.2007 P\right]$ where $P=\left(F_{\mathrm{o}}^{2}+2 F_{\mathrm{c}}^{2}\right) / 3$

$(\Delta / \sigma)_{\max }<0.001$

$\Delta \rho_{\max }=0.20 \mathrm{e}^{-3}$

$\Delta \rho_{\text {min }}=-0.21$ e $\AA^{-3}$

Extinction correction: SHELXL2014 (Sheldrick, $2015 b), \mathrm{Fc}^{*}=\mathrm{kFc}\left[1+0.001 \mathrm{xFc}^{2} \lambda^{3} / \sin (2 \theta)\right]^{-1 / 4}$

Extinction coefficient: 0.0164 (16) 


\section{Special details}

Geometry. All esds (except the esd in the dihedral angle between two 1.s. planes) are estimated using the full covariance matrix. The cell esds are taken into account individually in the estimation of esds in distances, angles and torsion angles; correlations between esds in cell parameters are only used when they are defined by crystal symmetry. An approximate (isotropic) treatment of cell esds is used for estimating esds involving l.s. planes.

Refinement. Refinement of $\mathrm{F}^{2}$ against ALL reflections. The weighted R-factor $\mathrm{wR}$ and goodness of fit $\mathrm{S}$ are based on $\mathrm{F}^{2}$, conventional R-factors $R$ are based on $F$, with $F$ set to zero for negative $\mathrm{F}^{2}$. The threshold expression of $\mathrm{F}^{2}>2 \operatorname{sigma}\left(\mathrm{F}^{2}\right)$ is used only for calculating R-factors(gt) etc. and is not relevant to the choice of reflections for refinement. R-factors based on $\mathrm{F}^{2}$ are statistically about twice as large as those based on F, and R- factors based on ALL data will be even larger.

Fractional atomic coordinates and isotropic or equivalent isotropic displacement parameters $\left(\hat{A}^{2}\right)$

\begin{tabular}{|c|c|c|c|c|}
\hline & $x$ & $y$ & $z$ & $U_{\text {iso }} * / U_{\text {eq }}$ \\
\hline $\mathrm{O} 1$ & $0.79721(16)$ & $0.27601(13)$ & $0.44040(10)$ & $0.0459(3)$ \\
\hline $\mathrm{O} 2$ & $0.93031(11)$ & $0.56110(10)$ & $0.87382(10)$ & $0.0299(3)$ \\
\hline N1 & $0.83593(16)$ & $0.38781(15)$ & 0.64465 (12) & $0.0344(3)$ \\
\hline $\mathrm{H} 1$ & $0.828(3)$ & $0.468(2)$ & $0.695(2)$ & $0.053(6)^{*}$ \\
\hline $\mathrm{N} 2$ & $0.77023(13)$ & $0.38862(11)$ & $0.89936(10)$ & $0.0226(3)$ \\
\hline $\mathrm{H} 2 \mathrm{~A}$ & $0.693(2)$ & $0.3631(18)$ & 0.9497 (18) & $0.037(5)^{*}$ \\
\hline N3 & $0.47391(14)$ & 0.69851 (11) & $0.95462(11)$ & $0.0266(3)$ \\
\hline N4 & $0.42223(14)$ & $0.79751(12)$ & $0.89372(11)$ & $0.0275(3)$ \\
\hline $\mathrm{H} 4 \mathrm{~A}$ & $0.315(3)$ & $0.798(2)$ & $0.870(2)$ & $0.050(6)^{*}$ \\
\hline $\mathrm{C} 1$ & $0.73450(18)$ & $0.51188(18)$ & $0.48918(14)$ & $0.0354(4)$ \\
\hline $\mathrm{C} 2$ & $0.7643(2)$ & $0.63951(19)$ & $0.56712(16)$ & $0.0421(4)$ \\
\hline $\mathrm{H} 2$ & $0.830(2)$ & $0.651(2)$ & $0.648(2)$ & $0.050(5)^{*}$ \\
\hline $\mathrm{C} 3$ & $0.7021(2)$ & $0.7553(2)$ & $0.53373(19)$ & $0.0514(5)$ \\
\hline H3 & $0.725(3)$ & $0.844(3)$ & $0.588(2)$ & $0.067(7)^{*}$ \\
\hline $\mathrm{C} 4$ & $0.6082(2)$ & $0.7447(2)$ & 0.42178 (19) & $0.0541(5)$ \\
\hline $\mathrm{H} 4$ & $0.569(3)$ & $0.827(2)$ & $0.402(2)$ & $0.062(6)^{*}$ \\
\hline $\mathrm{C} 5$ & $0.5786(2)$ & $0.6188(2)$ & $0.34362(17)$ & $0.0499(5)$ \\
\hline H5 & $0.509(3)$ & $0.608(2)$ & $0.264(2)$ & $0.055(6)^{*}$ \\
\hline C6 & 0.6409 (2) & $0.5025(2)$ & $0.37623(15)$ & $0.0433(4)$ \\
\hline H6 & $0.619(3)$ & $0.408(2)$ & $0.321(2)$ & $0.055(6)^{*}$ \\
\hline $\mathrm{C} 7$ & $0.79287(18)$ & $0.38224(17)$ & $0.52099(14)$ & $0.0352(4)$ \\
\hline $\mathrm{C} 8$ & $0.88775(17)$ & $0.27928(15)$ & $0.70092(13)$ & $0.0308(3)$ \\
\hline C9 & $0.9735(2)$ & $0.17147(18)$ & $0.63698(16)$ & $0.0416(4)$ \\
\hline H9 & $0.996(3)$ & $0.177(2)$ & $0.552(2)$ & $0.057(6)^{*}$ \\
\hline $\mathrm{C} 10$ & $1.0224(2)$ & $0.06677(18)$ & 0.69444 (16) & $0.0411(4)$ \\
\hline H10 & $1.082(2)$ & $-0.006(2)$ & $0.646(2)$ & $0.051(6)^{*}$ \\
\hline C11 & $0.98655(19)$ & $0.06710(15)$ & $0.81653(15)$ & $0.0348(3)$ \\
\hline H11 & $1.020(2)$ & $-0.006(2)$ & $0.858(2)$ & $0.050(6)^{*}$ \\
\hline $\mathrm{C} 12$ & $0.90333(17)$ & $0.17445(14)$ & 0.88159 (14) & $0.0280(3)$ \\
\hline H12 & $0.880(2)$ & $0.1783(17)$ & $0.9689(18)$ & $0.033(4)^{*}$ \\
\hline $\mathrm{C} 13$ & $0.85463(15)$ & $0.28126(13)$ & $0.82543(13)$ & $0.0247(3)$ \\
\hline C14 & $0.82037(15)$ & $0.51951(13)$ & $0.92651(12)$ & $0.0230(3)$ \\
\hline $\mathrm{C} 15$ & 0.73064 (17) & $0.61472(13)$ & $1.02316(13)$ & $0.0254(3)$ \\
\hline H15A & $0.810(2)$ & $0.6650(18)$ & $1.0828(18)$ & $0.035(4)^{*}$ \\
\hline H15B & $0.664(2)$ & $0.5616(18)$ & $1.0653(17)$ & $0.032(4)^{*}$ \\
\hline
\end{tabular}




$\begin{array}{lllll}\text { C16 } & 0.63237(16) & 0.71090(13) & 0.96200(13) & 0.0251(3) \\ \text { C17 } & 0.68045(17) & 0.81614(14) & 0.90528(15) & 0.0305(3) \\ \text { H17 } & 0.793(2) & 0.8410(19) & 0.8959(18) & 0.039(5)^{*} \\ \text { C18 } & 0.54213(17) & 0.86907(14) & 0.86181(14) & 0.0279(3) \\ \text { C19 } & 0.5098(2) & 0.98073(17) & 0.79240(18) & 0.0390(4) \\ \text { H19A } & 0.445(3) & 1.052(3) & 0.838(3) & 0.079(8)^{*} \\ \text { H19B } & 0.609(3) & 1.019(2) & 0.772(2) & 0.064(7)^{*} \\ \text { H19C } & 0.445(3) & 0.947(2) & 0.714(2) & 0.052(6)^{*} \\ \text { O3 } & 0.89511(12) & 0.24005(10) & 0.20075(10) & 0.0295(3) \\ \text { H3A } & 0.872(3) & 0.263(3) & 0.278(3) & 0.074(8)^{*} \\ \text { H3B } & 0.946(3) & 0.315(3) & 0.181(2) & 0.061(6)^{*} \\ \end{array}$

Atomic displacement parameters $\left(\AA^{2}\right)$

\begin{tabular}{lllllll}
\hline & $U^{11}$ & $U^{22}$ & $U^{33}$ & $U^{12}$ & $U^{13}$ & $U^{23}$ \\
\hline $\mathrm{O} 1$ & $0.0582(7)$ & $0.0541(7)$ & $0.0246(6)$ & $0.0020(6)$ & $0.0032(5)$ & $0.0061(5)$ \\
$\mathrm{O} 2$ & $0.0293(5)$ & $0.0313(5)$ & $0.0310(5)$ & $-0.0026(4)$ & $0.0063(4)$ & $0.0097(4)$ \\
$\mathrm{N} 1$ & $0.0403(7)$ & $0.0429(7)$ & $0.0214(6)$ & $0.0066(6)$ & $0.0041(5)$ & $0.0091(5)$ \\
$\mathrm{N} 2$ & $0.0248(5)$ & $0.0240(5)$ & $0.0202(5)$ & $0.0007(4)$ & $0.0042(4)$ & $0.0061(4)$ \\
$\mathrm{N} 3$ & $0.0275(6)$ & $0.0259(6)$ & $0.0275(6)$ & $0.0019(4)$ & $0.0033(5)$ & $0.0074(4)$ \\
$\mathrm{N} 4$ & $0.0265(6)$ & $0.0270(6)$ & $0.0303(6)$ & $0.0021(5)$ & $0.0022(5)$ & $0.0090(5)$ \\
$\mathrm{C} 1$ & $0.0303(7)$ & $0.0556(10)$ & $0.0240(7)$ & $-0.0008(7)$ & $0.0050(6)$ & $0.0156(6)$ \\
$\mathrm{C} 2$ & $0.0413(9)$ & $0.0539(10)$ & $0.0349(9)$ & $-0.0047(7)$ & $-0.0044(7)$ & $0.0203(7)$ \\
C3 & $0.0563(11)$ & $0.0548(11)$ & $0.0477(11)$ & $-0.0014(9)$ & $0.0004(9)$ & $0.0228(9)$ \\
C4 & $0.0508(11)$ & $0.0746(14)$ & $0.0483(11)$ & $0.0143(10)$ & $0.0088(9)$ & $0.0368(10)$ \\
C5 & $0.0385(9)$ & $0.0839(14)$ & $0.0327(9)$ & $0.0126(9)$ & $0.0027(7)$ & $0.0245(9)$ \\
C6 & $0.0357(8)$ & $0.0713(12)$ & $0.0251(8)$ & $0.0046(8)$ & $0.0028(6)$ & $0.0151(7)$ \\
C7 & $0.0323(7)$ & $0.0530(9)$ & $0.0217(7)$ & $-0.0005(7)$ & $0.0055(6)$ & $0.0099(6)$ \\
C8 & $0.0311(7)$ & $0.0371(8)$ & $0.0245(7)$ & $0.0046(6)$ & $0.0032(6)$ & $0.0069(6)$ \\
C9 & $0.0448(9)$ & $0.0514(10)$ & $0.0279(8)$ & $0.0117(8)$ & $0.0096(7)$ & $0.0041(7)$ \\
C10 & $0.0412(8)$ & $0.0415(9)$ & $0.0370(9)$ & $0.0115(7)$ & $0.0082(7)$ & $-0.0023(7)$ \\
C11 & $0.0359(8)$ & $0.0288(7)$ & $0.0389(8)$ & $0.0034(6)$ & $0.0042(7)$ & $0.0047(6)$ \\
C12 & $0.0297(7)$ & $0.0266(7)$ & $0.0279(7)$ & $-0.0002(5)$ & $0.0041(6)$ & $0.0055(5)$ \\
C13 & $0.0237(6)$ & $0.0267(6)$ & $0.0226(7)$ & $0.0000(5)$ & $0.0023(5)$ & $0.0026(5)$ \\
C14 & $0.0241(6)$ & $0.0264(6)$ & $0.0200(6)$ & $0.0016(5)$ & $-0.0007(5)$ & $0.0089(5)$ \\
C15 & $0.0297(7)$ & $0.0237(6)$ & $0.0234(7)$ & $0.0021(5)$ & $0.0023(6)$ & $0.0063(5)$ \\
C16 & $0.0276(6)$ & $0.0218(6)$ & $0.0253(7)$ & $0.0007(5)$ & $0.0016(5)$ & $0.0036(5)$ \\
C17 & $0.0275(7)$ & $0.0279(7)$ & $0.0386(8)$ & $-0.0009(6)$ & $0.0015(6)$ & $0.0128(6)$ \\
C18 & $0.0307(7)$ & $0.0240(6)$ & $0.0297(7)$ & $-0.0011(5)$ & $0.0024(6)$ & $0.0071(5)$ \\
C19 & $0.0421(9)$ & $0.0328(8)$ & $0.0464(10)$ & $0.0036(7)$ & $-0.0005(8)$ & $0.0191(7)$ \\
O3 & $0.0316(5)$ & $0.0291(5)$ & $0.0289(6)$ & $-0.0006(4)$ & $0.0034(4)$ & $0.0082(4)$ \\
& & & & & & \\
\hline & & & & & &
\end{tabular}

Geometric parameters $\left(A,{ }^{\circ}\right)$

\begin{tabular}{llll}
\hline $\mathrm{O} 1-\mathrm{C} 7$ & $1.236(2)$ & $\mathrm{C} 8-\mathrm{C} 9$ & $1.397(2)$ \\
$\mathrm{O} 2-\mathrm{C} 14$ & $1.2368(16)$ & $\mathrm{C} 8-\mathrm{C} 13$ & $1.399(2)$ \\
$\mathrm{N} 1-\mathrm{C} 7$ & $1.3578(19)$ & $\mathrm{C} 9-\mathrm{C} 10$ & $1.380(3)$ \\
$\mathrm{N} 1-\mathrm{C} 8$ & $1.413(2)$ & $\mathrm{C} 9-\mathrm{H} 9$ & $0.97(2)$
\end{tabular}




\begin{tabular}{|c|c|c|c|}
\hline N1-H1 & $0.88(2)$ & $\mathrm{C} 10-\mathrm{C} 11$ & $1.382(2)$ \\
\hline $\mathrm{N} 2-\mathrm{C} 14$ & $1.3444(17)$ & $\mathrm{C} 10-\mathrm{H} 10$ & $0.97(2)$ \\
\hline $\mathrm{N} 2-\mathrm{C} 13$ & $1.4316(17)$ & $\mathrm{C} 11-\mathrm{C} 12$ & $1.386(2)$ \\
\hline $\mathrm{N} 2-\mathrm{H} 2 \mathrm{~A}$ & $0.95(2)$ & C11-H11 & $0.98(2)$ \\
\hline $\mathrm{N} 3-\mathrm{C} 16$ & $1.3346(18)$ & $\mathrm{C} 12-\mathrm{C} 13$ & $1.390(2)$ \\
\hline N3-N4 & $1.3611(16)$ & $\mathrm{C} 12-\mathrm{H} 12$ & $0.978(19)$ \\
\hline $\mathrm{N} 4-\mathrm{C} 18$ & $1.3414(19)$ & $\mathrm{C} 14-\mathrm{C} 15$ & $1.5167(18)$ \\
\hline $\mathrm{N} 4-\mathrm{H} 4 \mathrm{~A}$ & $0.92(2)$ & $\mathrm{C} 15-\mathrm{C} 16$ & $1.5026(19)$ \\
\hline $\mathrm{C} 1-\mathrm{C} 2$ & $1.393(3)$ & C15-H15A & 0.957 (19) \\
\hline $\mathrm{C} 1-\mathrm{C} 6$ & $1.398(2)$ & C15-H15B & $0.972(19)$ \\
\hline $\mathrm{C} 1-\mathrm{C} 7$ & $1.492(2)$ & $\mathrm{C} 16-\mathrm{C} 17$ & $1.400(2)$ \\
\hline $\mathrm{C} 2-\mathrm{C} 3$ & $1.383(3)$ & $\mathrm{C} 17-\mathrm{C} 18$ & $1.377(2)$ \\
\hline $\mathrm{C} 2-\mathrm{H} 2$ & $0.99(2)$ & C17-H17 & 0.997 (19) \\
\hline $\mathrm{C} 3-\mathrm{C} 4$ & $1.388(3)$ & $\mathrm{C} 18-\mathrm{C} 19$ & $1.492(2)$ \\
\hline $\mathrm{C} 3-\mathrm{H} 3$ & $0.97(3)$ & C19-H19A & $0.98(3)$ \\
\hline $\mathrm{C} 4-\mathrm{C} 5$ & $1.380(3)$ & C19-H19B & $0.97(3)$ \\
\hline $\mathrm{C} 4-\mathrm{H} 4$ & $0.96(2)$ & $\mathrm{C} 19-\mathrm{H} 19 \mathrm{C}$ & $0.98(2)$ \\
\hline $\mathrm{C} 5-\mathrm{C} 6$ & $1.385(3)$ & $\mathrm{O} 3-\mathrm{H} 3 \mathrm{~A}$ & $0.87(3)$ \\
\hline $\mathrm{C} 5-\mathrm{H} 5$ & $1.00(2)$ & $\mathrm{O} 3-\mathrm{H} 3 \mathrm{~B}$ & $0.94(3)$ \\
\hline $\mathrm{C} 6-\mathrm{H} 6$ & $1.02(2)$ & & \\
\hline $\mathrm{C} 7-\mathrm{N} 1-\mathrm{C} 8$ & $127.23(14)$ & $\mathrm{C} 9-\mathrm{C} 10-\mathrm{C} 11$ & $120.29(14)$ \\
\hline $\mathrm{C} 7-\mathrm{N} 1-\mathrm{H} 1$ & $115.9(14)$ & $\mathrm{C} 9-\mathrm{C} 10-\mathrm{H} 10$ & $117.5(13)$ \\
\hline $\mathrm{C} 8-\mathrm{N} 1-\mathrm{H} 1$ & $116.9(14)$ & $\mathrm{C} 11-\mathrm{C} 10-\mathrm{H} 10$ & $122.2(13)$ \\
\hline $\mathrm{C} 14-\mathrm{N} 2-\mathrm{C} 13$ & $123.60(11)$ & $\mathrm{C} 10-\mathrm{C} 11-\mathrm{C} 12$ & $119.52(15)$ \\
\hline $\mathrm{C} 14-\mathrm{N} 2-\mathrm{H} 2 \mathrm{~A}$ & $117.1(11)$ & $\mathrm{C} 10-\mathrm{C} 11-\mathrm{H} 11$ & $121.3(12)$ \\
\hline $\mathrm{C} 13-\mathrm{N} 2-\mathrm{H} 2 \mathrm{~A}$ & $117.0(11)$ & $\mathrm{C} 12-\mathrm{C} 11-\mathrm{H} 11$ & $119.2(12)$ \\
\hline $\mathrm{C} 16-\mathrm{N} 3-\mathrm{N} 4$ & $104.44(11)$ & $\mathrm{C} 11-\mathrm{C} 12-\mathrm{C} 13$ & $120.87(14)$ \\
\hline $\mathrm{C} 18-\mathrm{N} 4-\mathrm{N} 3$ & $112.75(11)$ & $\mathrm{C} 11-\mathrm{C} 12-\mathrm{H} 12$ & $121.0(10)$ \\
\hline $\mathrm{C} 18-\mathrm{N} 4-\mathrm{H} 4 \mathrm{~A}$ & $129.2(13)$ & $\mathrm{C} 13-\mathrm{C} 12-\mathrm{H} 12$ & $118.1(10)$ \\
\hline $\mathrm{N} 3-\mathrm{N} 4-\mathrm{H} 4 \mathrm{~A}$ & $117.3(13)$ & $\mathrm{C} 12-\mathrm{C} 13-\mathrm{C} 8$ & $119.56(13)$ \\
\hline $\mathrm{C} 2-\mathrm{C} 1-\mathrm{C} 6$ & $118.94(16)$ & $\mathrm{C} 12-\mathrm{C} 13-\mathrm{N} 2$ & $117.38(12)$ \\
\hline $\mathrm{C} 2-\mathrm{C} 1-\mathrm{C} 7$ & $123.71(14)$ & $\mathrm{C} 8-\mathrm{C} 13-\mathrm{N} 2$ & $123.06(13)$ \\
\hline $\mathrm{C} 6-\mathrm{C} 1-\mathrm{C} 7$ & $117.33(16)$ & $\mathrm{O} 2-\mathrm{C} 14-\mathrm{N} 2$ & $122.29(12)$ \\
\hline $\mathrm{C} 3-\mathrm{C} 2-\mathrm{C} 1$ & $120.71(16)$ & $\mathrm{O} 2-\mathrm{C} 14-\mathrm{C} 15$ & $121.70(12)$ \\
\hline $\mathrm{C} 3-\mathrm{C} 2-\mathrm{H} 2$ & $117.6(12)$ & $\mathrm{N} 2-\mathrm{C} 14-\mathrm{C} 15$ & $115.99(12)$ \\
\hline $\mathrm{C} 1-\mathrm{C} 2-\mathrm{H} 2$ & $121.7(12)$ & $\mathrm{C} 16-\mathrm{C} 15-\mathrm{C} 14$ & $110.76(11)$ \\
\hline $\mathrm{C} 2-\mathrm{C} 3-\mathrm{C} 4$ & $119.9(2)$ & $\mathrm{C} 16-\mathrm{C} 15-\mathrm{H} 15 \mathrm{~A}$ & $109.7(11)$ \\
\hline $\mathrm{C} 2-\mathrm{C} 3-\mathrm{H} 3$ & $120.2(15)$ & $\mathrm{C} 14-\mathrm{C} 15-\mathrm{H} 15 \mathrm{~A}$ & $106.3(11)$ \\
\hline $\mathrm{C} 4-\mathrm{C} 3-\mathrm{H} 3$ & $119.9(15)$ & $\mathrm{C} 16-\mathrm{C} 15-\mathrm{H} 15 \mathrm{~B}$ & $110.7(10)$ \\
\hline $\mathrm{C} 5-\mathrm{C} 4-\mathrm{C} 3$ & $119.85(19)$ & $\mathrm{C} 14-\mathrm{C} 15-\mathrm{H} 15 \mathrm{~B}$ & $109.5(10)$ \\
\hline $\mathrm{C} 5-\mathrm{C} 4-\mathrm{H} 4$ & $123.0(15)$ & $\mathrm{H} 15 \mathrm{~A}-\mathrm{C} 15-\mathrm{H} 15 \mathrm{~B}$ & $109.7(15)$ \\
\hline $\mathrm{C} 3-\mathrm{C} 4-\mathrm{H} 4$ & $117.1(15)$ & N3-C16-C17 & $110.92(12)$ \\
\hline $\mathrm{C} 4-\mathrm{C} 5-\mathrm{C} 6$ & $120.57(16)$ & $\mathrm{N} 3-\mathrm{C} 16-\mathrm{C} 15$ & $119.15(12)$ \\
\hline $\mathrm{C} 4-\mathrm{C} 5-\mathrm{H} 5$ & $121.4(12)$ & $\mathrm{C} 17-\mathrm{C} 16-\mathrm{C} 15$ & $129.92(13)$ \\
\hline $\mathrm{C} 6-\mathrm{C} 5-\mathrm{H} 5$ & $118.0(13)$ & $\mathrm{C} 18-\mathrm{C} 17-\mathrm{C} 16$ & $105.70(13)$ \\
\hline $\mathrm{C} 5-\mathrm{C} 6-\mathrm{C} 1$ & $120.03(18)$ & $\mathrm{C} 18-\mathrm{C} 17-\mathrm{H} 17$ & $129.2(11)$ \\
\hline $\mathrm{C} 5-\mathrm{C} 6-\mathrm{H} 6$ & $121.8(12)$ & $\mathrm{C} 16-\mathrm{C} 17-\mathrm{H} 17$ & $125.0(11)$ \\
\hline
\end{tabular}




\begin{tabular}{|c|c|c|c|}
\hline $\mathrm{C} 1-\mathrm{C} 6-\mathrm{H} 6$ & $118.1(13)$ & $\mathrm{N} 4-\mathrm{C} 18-\mathrm{C} 17$ & $106.19(12)$ \\
\hline $\mathrm{O} 1-\mathrm{C} 7-\mathrm{N} 1$ & $122.37(16)$ & $\mathrm{N} 4-\mathrm{C} 18-\mathrm{C} 19$ & $120.86(13)$ \\
\hline $\mathrm{O} 1-\mathrm{C} 7-\mathrm{C} 1$ & $122.02(14)$ & $\mathrm{C} 17-\mathrm{C} 18-\mathrm{C} 19$ & $132.95(14)$ \\
\hline $\mathrm{N} 1-\mathrm{C} 7-\mathrm{C} 1$ & $115.58(14)$ & C18-C19-H19A & $112.3(16)$ \\
\hline $\mathrm{C} 9-\mathrm{C} 8-\mathrm{C} 13$ & $118.99(14)$ & $\mathrm{C} 18-\mathrm{C} 19-\mathrm{H} 19 \mathrm{~B}$ & $110.6(14)$ \\
\hline $\mathrm{C} 9-\mathrm{C} 8-\mathrm{N} 1$ & $122.09(14)$ & $\mathrm{H} 19 \mathrm{~A}-\mathrm{C} 19-\mathrm{H} 19 \mathrm{~B}$ & $111(2)$ \\
\hline $\mathrm{C} 13-\mathrm{C} 8-\mathrm{N} 1$ & $118.91(13)$ & $\mathrm{C} 18-\mathrm{C} 19-\mathrm{H} 19 \mathrm{C}$ & $110.7(12)$ \\
\hline $\mathrm{C} 10-\mathrm{C} 9-\mathrm{C} 8$ & $120.75(15)$ & H19A-C19-H19C & $104(2)$ \\
\hline $\mathrm{C} 10-\mathrm{C} 9-\mathrm{H} 9$ & $123.7(13)$ & $\mathrm{H} 19 \mathrm{~B}-\mathrm{C} 19-\mathrm{H} 19 \mathrm{C}$ & $108.2(19)$ \\
\hline $\mathrm{C} 8-\mathrm{C} 9-\mathrm{H} 9$ & $115.5(13)$ & $\mathrm{H} 3 \mathrm{~A}-\mathrm{O} 3-\mathrm{H} 3 \mathrm{~B}$ & $107(2)$ \\
\hline $\mathrm{C} 16-\mathrm{N} 3-\mathrm{N} 4-\mathrm{C} 18$ & $0.84(15)$ & $\mathrm{C} 11-\mathrm{C} 12-\mathrm{C} 13-\mathrm{C} 8$ & $1.1(2)$ \\
\hline $\mathrm{C} 6-\mathrm{C} 1-\mathrm{C} 2-\mathrm{C} 3$ & $0.1(3)$ & $\mathrm{C} 11-\mathrm{C} 12-\mathrm{C} 13-\mathrm{N} 2$ & $-179.58(13)$ \\
\hline $\mathrm{C} 7-\mathrm{C} 1-\mathrm{C} 2-\mathrm{C} 3$ & $-177.84(16)$ & $\mathrm{C} 9-\mathrm{C} 8-\mathrm{C} 13-\mathrm{C} 12$ & $-1.9(2)$ \\
\hline $\mathrm{C} 1-\mathrm{C} 2-\mathrm{C} 3-\mathrm{C} 4$ & $0.3(3)$ & $\mathrm{N} 1-\mathrm{C} 8-\mathrm{C} 13-\mathrm{C} 12$ & $179.30(13)$ \\
\hline $\mathrm{C} 2-\mathrm{C} 3-\mathrm{C} 4-\mathrm{C} 5$ & $-0.6(3)$ & $\mathrm{C} 9-\mathrm{C} 8-\mathrm{C} 13-\mathrm{N} 2$ & $178.78(14)$ \\
\hline $\mathrm{C} 3-\mathrm{C} 4-\mathrm{C} 5-\mathrm{C} 6$ & $0.4(3)$ & $\mathrm{N} 1-\mathrm{C} 8-\mathrm{C} 13-\mathrm{N} 2$ & $0.0(2)$ \\
\hline $\mathrm{C} 4-\mathrm{C} 5-\mathrm{C} 6-\mathrm{C} 1$ & $0.1(3)$ & $\mathrm{C} 14-\mathrm{N} 2-\mathrm{C} 13-\mathrm{C} 12$ & $120.82(14)$ \\
\hline $\mathrm{C} 2-\mathrm{C} 1-\mathrm{C} 6-\mathrm{C} 5$ & $-0.4(2)$ & $\mathrm{C} 14-\mathrm{N} 2-\mathrm{C} 13-\mathrm{C} 8$ & $-59.84(18)$ \\
\hline $\mathrm{C} 7-\mathrm{C} 1-\mathrm{C} 6-\mathrm{C} 5$ & $177.75(15)$ & $\mathrm{C} 13-\mathrm{N} 2-\mathrm{C} 14-\mathrm{O} 2$ & $11.49(19)$ \\
\hline $\mathrm{C} 8-\mathrm{N} 1-\mathrm{C} 7-\mathrm{O} 1$ & $-1.2(3)$ & $\mathrm{C} 13-\mathrm{N} 2-\mathrm{C} 14-\mathrm{C} 15$ & $-170.30(11)$ \\
\hline $\mathrm{C} 8-\mathrm{N} 1-\mathrm{C} 7-\mathrm{C} 1$ & $176.82(14)$ & $\mathrm{O} 2-\mathrm{C} 14-\mathrm{C} 15-\mathrm{C} 16$ & $68.48(16)$ \\
\hline $\mathrm{C} 2-\mathrm{C} 1-\mathrm{C} 7-\mathrm{O} 1$ & $-163.00(17)$ & $\mathrm{N} 2-\mathrm{C} 14-\mathrm{C} 15-\mathrm{C} 16$ & $-109.73(13)$ \\
\hline $\mathrm{C} 6-\mathrm{C} 1-\mathrm{C} 7-\mathrm{O} 1$ & $19.0(2)$ & N4-N3-C16-C17 & $-0.49(15)$ \\
\hline $\mathrm{C} 2-\mathrm{C} 1-\mathrm{C} 7-\mathrm{N} 1$ & $19.0(2)$ & $\mathrm{N} 4-\mathrm{N} 3-\mathrm{C} 16-\mathrm{C} 15$ & $-179.14(11)$ \\
\hline $\mathrm{C} 6-\mathrm{C} 1-\mathrm{C} 7-\mathrm{N} 1$ & $-159.01(14)$ & $\mathrm{C} 14-\mathrm{C} 15-\mathrm{C} 16-\mathrm{N} 3$ & $111.39(14)$ \\
\hline $\mathrm{C} 7-\mathrm{N} 1-\mathrm{C} 8-\mathrm{C} 9$ & $32.0(2)$ & $\mathrm{C} 14-\mathrm{C} 15-\mathrm{C} 16-\mathrm{C} 17$ & $-66.96(18)$ \\
\hline $\mathrm{C} 7-\mathrm{N} 1-\mathrm{C} 8-\mathrm{C} 13$ & $-149.26(15)$ & N3-C16-C17-C18 & $0.00(16)$ \\
\hline $\mathrm{C} 13-\mathrm{C} 8-\mathrm{C} 9-\mathrm{C} 10$ & $1.3(3)$ & $\mathrm{C} 15-\mathrm{C} 16-\mathrm{C} 17-\mathrm{C} 18$ & $178.46(13)$ \\
\hline $\mathrm{N} 1-\mathrm{C} 8-\mathrm{C} 9-\mathrm{C} 10$ & $-179.91(15)$ & $\mathrm{N} 3-\mathrm{N} 4-\mathrm{C} 18-\mathrm{C} 17$ & $-0.86(16)$ \\
\hline $\mathrm{C} 8-\mathrm{C} 9-\mathrm{C} 10-\mathrm{C} 11$ & $0.1(3)$ & $\mathrm{N} 3-\mathrm{N} 4-\mathrm{C} 18-\mathrm{C} 19$ & $178.99(13)$ \\
\hline $\mathrm{C} 9-\mathrm{C} 10-\mathrm{C} 11-\mathrm{C} 12$ & $-1.0(3)$ & $\mathrm{C} 16-\mathrm{C} 17-\mathrm{C} 18-\mathrm{N} 4$ & $0.50(16)$ \\
\hline $\mathrm{C} 10-\mathrm{C} 11-\mathrm{C} 12-\mathrm{C} 13$ & $0.4(2)$ & $\mathrm{C} 16-\mathrm{C} 17-\mathrm{C} 18-\mathrm{C} 19$ & $-179.31(16)$ \\
\hline
\end{tabular}

Hydrogen-bond geometry $\left(A,{ }^{\circ}\right)$

$\mathrm{Cg} 1$ and $\mathrm{Cg} 3$ are the centroids of the $\mathrm{N} 3, \mathrm{~N} 4, \mathrm{C} 16-\mathrm{C} 18$ and $\mathrm{C} 8-\mathrm{C} 13$ rings respectively.

\begin{tabular}{lllll}
\hline$D-\mathrm{H} \cdots A$ & $D-\mathrm{H}$ & $\mathrm{H} \cdots A$ & $D \cdots A$ & $D-\mathrm{H} \cdots A$ \\
\hline $\mathrm{N} 1-\mathrm{H} 1 \cdots \mathrm{O} 2$ & $0.88(2)$ & $2.09(2)$ & $2.7902(17)$ & $135.0(19)$ \\
$\mathrm{N} 2-\mathrm{H} 2 A \cdots \mathrm{N} 3^{\mathrm{i}}$ & $0.95(2)$ & $1.98(2)$ & $2.9209(17)$ & $176.4(17)$ \\
$\mathrm{N} 4-\mathrm{H} 4 A \cdots \mathrm{O} 3^{\mathrm{ii}}$ & $0.92(2)$ & $1.88(2)$ & $2.7883(16)$ & $166.9(19)$ \\
$\mathrm{C} 12-\mathrm{H} 12 \cdots \mathrm{O} 3^{\mathrm{iii}}$ & $0.978(19)$ & $2.464(19)$ & $3.4042(19)$ & $161.2(14)$ \\
$\mathrm{O} 3-\mathrm{H} 3 A \cdots \mathrm{O} 1$ & $0.87(3)$ & $1.89(3)$ & $2.7445(16)$ & $167(2)$ \\
$\mathrm{O} 3-\mathrm{H} 3 B \cdots \mathrm{O} 2^{\mathrm{iv}}$ & $0.94(3)$ & $1.83(3)$ & $2.7575(15)$ & $169(2)$ \\
$\mathrm{C} 15-\mathrm{H} 15 A \cdots C g 3^{\mathrm{v}}$ & $0.957(19)$ & $2.893(18)$ & $3.8485(15)$ & $174.0(14)$ \\
$\mathrm{C} 19-\mathrm{H} 19 A \cdots C g 1^{\mathrm{vi}}$ & $0.98(3)$ & $2.86(3)$ & $3.645(2)$ & $138(2)$
\end{tabular}

Symmetry codes: (i) $-x+1,-y+1,-z+2$; (ii) $-x+1,-y+1,-z+1$; (iii) $x, y, z+1$; (iv) $-x+2,-y+1,-z+1$; (v) $-x+2,-y+1,-z+2$; (vi) $-x+1,-y+2,-z+2$. 